Nuria Andreu · Maricruz García-Rodríguez

Victor Volpini · Cecilia Frecha · Ignacio J. Molina

Gumersindo Fontan · Cristina Fillat

\title{
A novel Wiskott-Aldrich syndrome protein (WASP) complex mutation identified in a WAS patient results in an aberrant product at the C-terminus from two transcripts with unusual polyA signals
}

Received: 28 July 2005 / Accepted: 7 October 2005/Published online: 22 December 2005

(C) The Japan Society of Human Genetics and Springer-Verlag 2005

\begin{abstract}
Wiskott-Aldrich syndrome (WAS) is an $\mathrm{X}$-linked recessive disorder characterized by immunodeficiency, thrombocytopenia and eczema. A broad spectrum of mutations in the WASP gene has been identified as causing the disease. In the present paper, we report on a patient affected by WAS with a novel complex mutation, characterized by a small 9 bp deletion followed by an inversion of $151 \mathrm{bp}$ and a gross deletion of $4.3 \mathrm{~kb}$ within the Xp11.23 region. The small deletion and the inverted fragment are found in intron 11 . The large deletion initiates downstream of exon 11 of the WASP gene, including exon 12, and a genomic region upstream of the promoter of the contiguous SUV39H1 gene. Expression studies of the mRNA of the patient's sample showed the presence of two aberrant transcripts that code for a protein of 519 amino acids. We demonstrate that these two transcripts differ in the 3' UTR region, and result from the use of two alternative polyadenylation signals. The severe phenotype of the patient correlates with the presence of an aberrant protein.
\end{abstract}

Keywords Wiskott-Aldrich syndrome - WASP · Mutation · Deletion · Inversion

N. Andreu · C. Fillat $(\bowtie)$

Programa Gens i Malaltia, Centre de Regulació Genòmica-CRGUPF, Passeig Marítim, 37-49, 08003 Barcelona, Spain

E-mail: cristina.fillat@crg.es

Tel.: + 34-93-2240942

Fax: + 34-93-2240899

M. García-Rodríguez · G. Fontan

Unidad de Inmunología, Hospital Universitario la Paz,

Madrid, Spain

V. Volpini

Centre de Diagnosi Genètic Molecular-IRO-IDIBELL,

L'Hospitalet de Llobregat, Spain

C. Frecha $\cdot$ I.J. Molina

Unidad de Inmunología, Instituto de Biopatología y Medicina Regenarativa, Facultad de Medicina, Universidad de Granada, Granada, Spain

\section{Introduction}

Wiskott-Aldrich syndrome (WAS) is an X-linked recessive immune deficiency disorder (MIM\#301000) characterized by thrombocytopenia with small platelets, eczema and recurrent infections. It mostly affects boys, although several cases of female WAS have also been reported (Parolini et al. 1998; Inoue et al. 2002; Lutskiy et al. 2002; Andreu et al. 2003b). The gene involved in the disease is located on the short arm of the $\mathrm{X}$ chromosome in region $\mathrm{Xp11.22-23}$ at $5 \mathrm{~kb}$ from the SUV39H1 or human homolog of $\mathrm{Su}(\mathrm{var}) 3-9$, a gene involved in the regulation of higher order chromatin organization (Hagemann et al. 2000). Mutations in the Wiskott-Aldrich syndrome protein (WASP) gene, leading to a loss of function, have been described in patients with WAS and X-linked thrombocytopenia (XLT) (Derry et al. 1994; Zhu et al. 1995; Ochs and Rosen 1999). Gain of function mutations in the same gene have been found to cause X-linked severe congenital neutropenia (Devriendt et al. 2001). The WASP gene has 12 exons and encodes for a cytoplasmatic protein (WASP), predominantly expressed in hematopoietic cells, and it plays a key role in regulating changes in cytoskeletal structure in response to external stimuli and signal transduction processes (Brickell et al. 1998; Snapper and Rosen 1999).

Approximately 300 unique mutations have been reported in the WASP gene, while only five mutational hotspots have been clearly identified (Imai et al. 2004; Jin et al. 2004) (http://homepage.mac.com/kohsukeimai/ wasp/WASPbase.html). Mutations are found throughout the entire gene, with missense mutations preferentially locating in the amino-terminal part of the protein, and stop and frameshift mutations mainly in the carboxyl-terminal (Schindelhauer et al. 1996; Fillat et al. 2001). Mutations in the WASP gene cause a wide variety of clinical phenotypes, ranging from isolated thrombocytopenia to severe WAS. Phenotype/genotype correla- 
tion studies indicate that mutations resulting in the expression of normal-sized mutated protein are associated with a milder phenotype, while mutations leading to a truncated or absent protein correlate with a more severe phenotype (Snapper and Rosen 1999; Jin et al. 2004). However, of all the mutations reported, only approximately one-third involve WASP expression studies at the mRNA or protein level.

In the present study, we describe for the first time a complex WASP mutation that results in the expression of two alternative mRNA products, derived from two different polyadenylation signals. At the genomic level, the mutation involves a small $9 \mathrm{bp}$ deletion followed by a $151 \mathrm{bp}$ inversion and a large deletion of $4.3 \mathrm{~Kb}$, involving intron 11, exon 12 and the $3^{\prime} \mathrm{UTR}$ of the WASP gene, and the genomic region upstream of the SUV39H1 gene.

\section{Materials and methods}

\section{Case report}

The propositus is a boy clinically diagnosed as having Wiskott-Aldrich syndrome at 3.5 months of age. On the second day of his life he was already presenting bloody stools. When only 18 days old, he suffered a urinary tract infection and presented anaemia, lymphopenia and thrombocytopenia. At two months of age he developed generalized dermatitis. Occasional bouts of oral candidiasis were observed. His platelet count varied from 17 to $28 \times 10^{3}$ cells $/ \mathrm{mm}^{3}$, with a normal mean volume, although when observed under light microscopy, both micro- and macrothrombocytes were identified. Immunological studies revealed a decrease in absolute lymphocyte number $\left(2.200-3.270\right.$ cells $\left./ \mathrm{mm}^{3}\right)$, with low CD3 + cells $\left(325-580\right.$ cells $\left./ \mathrm{mm}^{3}\right)$. This CD3 lymphopenia affected both CD4 + and CD8 + cells. However, B and NK cell values were normal. Serum immunoglobulins were also found to be normal. Evaluation of T-cell function indicated a decrease in T-cell proliferation in response to mitogenic stimuli (phytohemagglutinin, concanavalin A, pokeweed mitogen and OKT3 monoclonal antibody anti-CD3). Other types of primary immunodeficiencies, such as adenosine deaminase (ADA) and purine nucleoside phosphorylase (PNP) deficiencies were excluded as the pertinent enzyme activity in erythrocytes was found to be normal, and no changes in the sequences of the common $\gamma$ chain, the $\alpha$ chain of the IL7 receptor or Jak3 genes were detected. An allogeneic bone marrow transplant from his HLA-identical sister was performed at the age of ten months. During the post-transplantation period he suffered HV-6 and CMV infections. He is presently 24 months old and has not suffered recurrent infections, but does present psychomotor retardation of unknown etiology. The number and subpopulations of lymphocytes, platelets and neutrophils were found to be normal, and his peripheral blood cells presented $100 \%$ chimerism.
Screening for WASP mutation

Informed consent was obtained to perform WASP mutational analysis in blood samples taken from both the patient and his mother. DNA was extracted from peripheral blood leukocytes by standard techniques. The WASP exons were amplified as previously described (Fillat et al. 2001). Exons 1-11 and the flanking splice site of the WASP gene were analyzed by SSCA (Fillat et al. 2001).

\section{Characterization of the breakpoint}

To localize the deletion breakpoint, we carried out multiple PCR amplification of nonoverlapping fragments by designing four primer pairs spaced every 1-2 kb along the WASP and SUV39H1 genes. Two primer pairs amplified PCR products from the genomic region between the WASP and the SUV39H1 genes (PCR fragments $\mathrm{W}$ and $\mathrm{X}$ ). The other two primer pairs were used to amplify fragments from the SUV39H1 gene (PCR fragments $\mathrm{Y}$ and $\mathrm{Z}$ ). A complete list of the primers used is shown in Table 1.

To characterize the deletion breakpoint, we used a PCR approach, employing the following primers: E11W-L, which anneals to exon 11 of the WASP gene, and X-R, which anneals to the intergenic region. The PCR product was resolved on $2 \%$ agarose gel, purified with the PCR purification kit (Qiagen, Valencia, CA, USA) and DNA-sequenced with BigDye terminator chemistry (Applied Biosystems, Foster City, CA, USA). Mutation was confirmed in two independent polymerase chain reaction (PCR) amplifications.

\section{RT-PCR analysis}

An RT-PCR analysis was performed as previously described (Andreu et al. 2003a). Briefly, $1 \mu \mathrm{g}$ of total RNA from each sample was reverse-transcribed with a Retroscript RT kit (Ambion, Austin, TX, USA). Five microlitres of cDNA was PCR-amplified with WASPspecific primers (listed in Table 2). We performed different PCR reactions to amplify the WASP cDNA, using the

Table 1 Primers used to characterize the breakpoint

\begin{tabular}{|c|c|}
\hline Primer & Sequence \\
\hline W-F & 5'- TAACAACTCACACACCAGGA-3' \\
\hline W-R & 5'- ССССАСТАССТТТСТСТТТТ-3' \\
\hline$X-F$ & 5'- CAAAAATTAGCCAGGTGTG-3' \\
\hline $\mathrm{X}-\mathrm{R}$ & 5'- AGGCTGAGCAACCATTAAG-3' \\
\hline $\mathrm{Y}-\mathrm{F}$ & 5'- TAACGTGATTGGCTAGCAG-3' \\
\hline Y-R & 5'- АТАСТTТССССCAGAAGAG-3' \\
\hline $\mathrm{Z}-\mathrm{F}$ & 5'- CCCACAGCATATCACTTAC-3' \\
\hline Z-R & 5'- GTCAAGTCCAGTCAAAACC-3' \\
\hline E11W-L & $5^{\prime}$ - ATGCACGTGATGCAGAAGAG-3' \\
\hline
\end{tabular}


Table 2 Primers used for RT-PCR

\begin{tabular}{|c|c|c|}
\hline Primer & Sequence & Position \\
\hline W1S & 5'-GCCTCGCCAGAGAAGACAAG-3' & Exon 1 \\
\hline E6-7W-R & 5'- ACTGGAGGGCCTCCTTGGTC-3' & $\begin{array}{l}\text { Junction of } \\
\text { exon } 6 \text { and } 7\end{array}$ \\
\hline E4W-L & 5'- AAGGAATCAGAGGCAAAGTGG-3' & Exon 4 \\
\hline W10S2 & 5'-TCCAGCTACTGGACGTTCTG-3' & Exon 10 \\
\hline W10A1 & 5'-CATGGGTGGCCCACCAGCTC-3' & Exon 10 \\
\hline E11W-R & 5'- CTGCAGCGCTGAGCTCTCTG-3' & Exon 11 \\
\hline E11W-L & 5'- ATGCACGTGATGCAGAAGAG-3' & Exon 11 \\
\hline W12A & 5'- TGGAGGGGGAGCCATGTC-3' & $3^{\prime} \mathrm{UTR}$ \\
\hline
\end{tabular}

indicated primers. RT-PCR products from exon 1 to 7 ; exon 4 to 10 ; exon 10 to 11 ; exon 10 to 12 and exon 11 to 12 were amplified and run in a $2 \%$ agarose gel stained with ethidium bromide. All RT-PCR results were confirmed by two independent RT-PCR amplifications.

3' RACE (rapid amplification of cDNA end)

3' RACE synthesis of first-strand cDNA was performed according to the procedure previously described (Frohman 1990). Briefly, $1 \mu \mathrm{g}$ of total RNA was mixed with MMLV-Retrotranscriptase (Ambion) and the $5 \mu \mathrm{M}$ adaptor primer $3^{\prime}$ RACE-AS (5'-TAATACGA CTCACTATAGGGCGATCC (T) ${ }_{25} \mathrm{VN}-3^{\prime}$ ), under the conditions recommended by the manufacturer. One microlitre of the reaction product was amplified in a $25 \mu \mathrm{l}$ PCR reaction using the PCR enhancer system (Gibco, NY, USA). The primers used to amplify the 3'end of WASP cDNA were E10W-F2 $5^{\prime}$ GAGCGC TTTTGGATCAAATC $3^{\prime}$ and T7-AS 5' GTAATACG ACTCACTATAGGGC $3^{\prime}$. PCR products were purified by agarose gel electrophoresis and cloned in pGEM-T Easy Vector System I (Promega, Madison, WI, USA). Cloned cDNA fragments were then sequenced with the Big Dye terminator cycle sequencing kit (Applied Biosystems) and an ABIPRISM3100 genetic analyzer (Applied Biosystems).

\section{Western immunoblotting of WASP}

Blood peripheral lymphocytes from the patient and a healthy individual were isolated by Ficoll-Hypaque gradient centrifugation. WASP protein analysis was carried out as detailed elsewhere (Martin et al. 2005). Briefly, cells were lysed with 1\% NP-40 lysis buffer containing protease inhibitor cocktail (Sigma, St Louis, MO, USA), resolved by SDS-PAGE and electrotransferred to Hybond-P PVDF membranes (Amersham, Piscataway, NJ, USA). Membranes were blocked with $5 \%$ non-fat milk and probed for $1 \mathrm{~h}$ at RT with $1 \mu \mathrm{g} / \mathrm{ml}$ of the anti-WASP mAb D1 (Santa Cruz Biotechnology, Santa Cruz, CA, USA) followed by a similar incubation with HRPO-labeled goat anti-mouse antibody (1:10,000 dilution) (Caltag, Botolph Claydon, UK). The blot was developed by chemiluminescence (ECL, Amersham) and exposed to autoradiographs (Amersham).

\section{Results and discussion}

Using PCR analysis of genomic DNA, we identified a complex mutation in the Xp11.22-23 region of a patient clinically diagnosed with WAS (accession number AM085518). Exon amplification and SSCA analysis of exons 1 to 12 revealed that the patient's sample lacked exon 12, the last exon of the WASP gene. PCR amplification of four nonoverlapping DNA fragments (labeled $\mathrm{W}, \mathrm{X}, \mathrm{Y}$ and $\mathrm{Z}$ ), spanning the region between the WASP and SUV39H1 genes, showed that fragment W was not present in the patient's sample (Fig. 1a,b). Primer pairs E11W-L and X-R amplified a fragment containing the breakpoint of the large deletion $(4,356 \mathrm{bp})$. The deleted fragment involved $1,347 \mathrm{bp}$ of intron 11 , coding exon 12 and $3^{\prime}$ UTR of the WASP gene, as well as DNA sequences upstream of the promoter region of the contiguous SUV39H1 gene (Hagemann et al. 2000). Additionally, a small 9 bp deletion and an inversion of $151 \mathrm{bp}$, with an adenine insertion in nucleotide 148 of the inverted sequence were also identified (Fig. 1c). Family studies in the patient's mother showed that she was not a carrier for the mutation, indicating that this was a de novo mutation (data not shown).

Only a few complex mutations have been identified in WAS patients. In a recent study of 227 WAS/XLT families, only $10 \%$ of the total mutations studied could be classified as complex, with a broad spectrum of phenotypes (Jin et al. 2004). In the present study the mutation could be associated with a severe form of WAS presenting a clinical score of 4 (Zhu et al. 1995). Progress of the disease could not be studied because the patient underwent bone marrow transplantation at the age of ten months.

To explore the consequences of the mutation effect, expression studies were performed on the patient's RNA samples. RT-PCR analysis with the indicated primers showed that the WASP mRNA contained exons 1 to 11 of the WASP gene (Fig. 2a). In order to identify the complete open reading frame of the aberrant WASP mRNA transcript, we performed 3' RACE experiments. 
Interestingly, we detected the presence of two aberrant transcripts of lengths 767 and $557 \mathrm{bp}$, respectively (Fig. 2b,c). Sequence analysis of the two fragments showed that they were different in the $3^{\prime}$ UTR region, but had a putative common coding region. Each transcript used a particular polyadenylation signal, different to that of wild-type WASP. The polyA signal of wildtype WASP, located in exon 12, was absent in the patient's sample as a consequence of the mutation. Alternatively, the patient's mRNA used a polyA signal to generate the longer transcript characterized by the canonical hexanucleotide sequence AAUAAA located downstream of the inverted sequence. Interestingly, the second smaller transcript was produced from the novel polyA signal, generated as a consequence of the breakpoint sequence and the A insertion, corresponding to hexanucleotide AUUAAA (Fig. 2c,d). In the mammalian system, effective polyadenylation has been found to require two main sequence components: a highly conserved hexanucleotide signal located 10-30 nucleotides $5^{\prime}$ from the cleavage site, and a more variable GU element located 20-40 bases $3^{\prime}$ from the cleavage site. Several hexanucleotide variants have been identified in the human genome. The two variants identified in the transcripts of the WAS patient are the two signals most commonly found. In fact, the AAUAAA sequence is considered a canonical signal, and is found to be present in $90 \%$ of the mRNA, while the second most frequent variant AUUAAA is found in the remaining $10 \%$ (Colgan and Manley 1997; Beaudoing et al. 2000). Moreover, both signals have been reported to possess comparable polyadenylation activity (Zhao et al. 1999).

The two transcripts had the same open reading frame, with a stop codon in intron 11 . The aberrant mRNAs encoded for a hypothetical abnormal WASP protein of 519 amino acids. This protein is different to wild-type WASP (502 amino acids) in that it lacks 17 amino acids from exon 12, and includes 34 new amino acids from intron 11. Western blot analysis showed that this protein was expressed and apparently had a similar size to the wild-type protein (Fig. 3). A mutation leading to a protein of 519 amino acids has recently been described in three patients (Imai et al. 2004). However, this mutation was completely different from the one we describe in the present paper, in terms of both the genomic and mRNA changes. Interestingly our results demonstrate that the 519 amino acid protein, derived from two aberrant transcripts, is a stable protein. Nevertheless, the protein
Fig. 1a-c Mutation screening. a Schematic representation of WASP and SUV39H1 genes on chromosome Xp11. Structure of the WASP gene (or WASP genomic organization). Unshaded blocks represent uncoded regions. The 12 exons coding 502 amino acids are represented as boxes (and numbered) while intronic sequences are shown as thin bars. PCR fragments $(\mathrm{W}, \mathrm{X}, \mathrm{Y}$ and $Z$ ) are shown in the scheme. b Schematic diagram representing the affected region of the WASP gene. The arrow shows the inverted sequence. Square brackets represent deleted regions. c Wild-type and patient sequences of the affected region. Exon 11 is shown in bold. Deleted sequences are between brackets. In bold and italic type, inverted sequence (151 bp). Underlined, the A insertion a

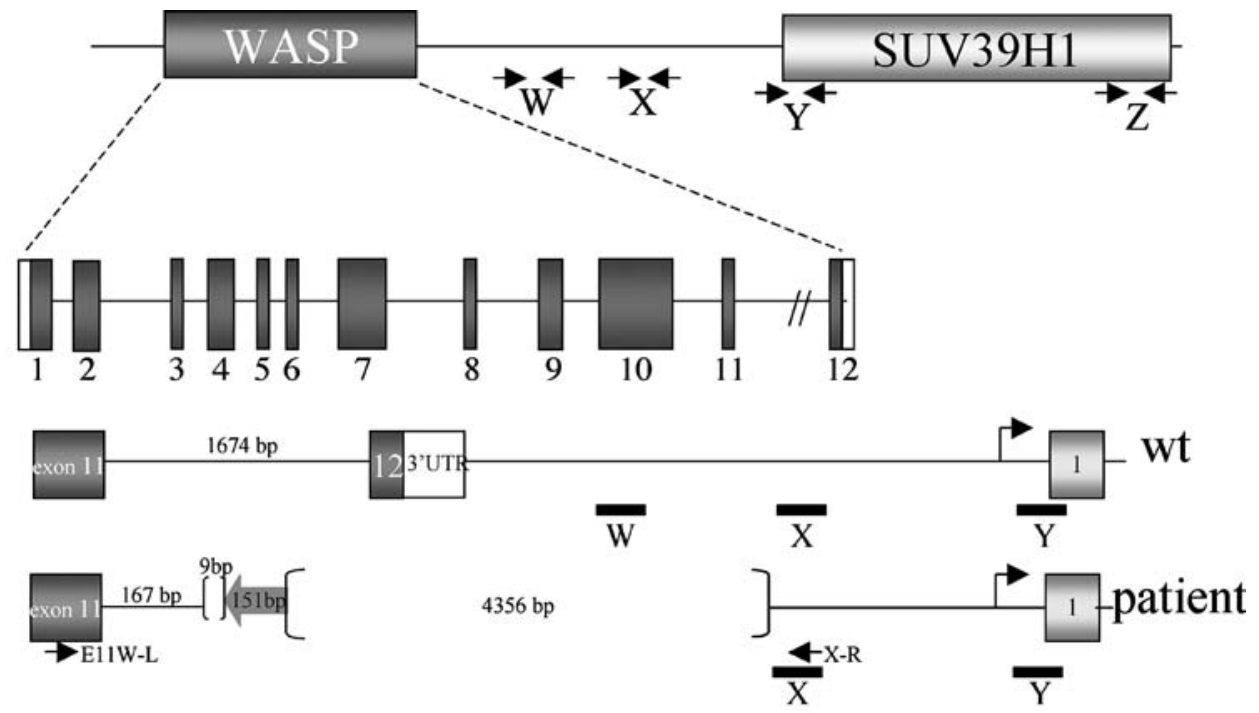

C Wild-type sequence

GATGCAGAAGAGAAGCAGAGCCATCCACTCCTCCGGTGAGCTGATCCTGCCGGGGCCTCAAACCTGGCTCCC AGGGCTAGCACTGGCCTCAAAACAATCCCAGCAGTCACCACCAATAGTGACATCAGCCCCATCTGTTTGACA GCATTAACATGAATCTTGTGTCAGCCTCGTTTTTGACAATGTTAACATTAAGTCATTA (TGTGACAAT) AAT ATAATTAACTCCAACTTTGACAGTAATATTAACATTAATGCCAGGGTGTGTCCACAATATTAATGTCATTCC CACATGTTCAGTACTACTAACATCAGCTGGCCGGGCGCGGTGGCTCATGCCTGTAATCCAGGAACTTTGGGA GGC (TAAGGCAGGA . . .4356 bp ... CTGCCTGGCC) AACGTGGTGAAACCCCATCATCTCTACTAAAAA Patient's sequence

GATGCAGAAGAGAAGCAGAGCCATCCACTCCTCCGGTGAGCTGATCCTGCCGGGGCCTCAAACCTGGCTCCC AGGGCTAGCACTGGCCTCAAAACAATCCCAGCAGTCACCACCAATAGTGACATCAGCCCCATCTGTTTGACA GCATTAACATGAATCTTGTGTCAGCCTCGTTTTTGACAATGTTAACATTAAGTCATTAGCCTCCCAAAGTTC CTGGATTACAGGCATGAGCCACCGCGCCCGGCCAGCTGATGTTAGTAGTACTGAACATGTGGGAATGACATT AATATTGTGGACACACCCTGGCATTAATGTTAATATTACTGTCAAAGTTGGAGTTAATTATAATTAACGTGG TGAAACCCCATCATCTCTACTAAAAA 
Fig. 2a-d Expression of the WASP gene. a Diagram of WASP mRNA and a table summarizing RT-PCR results. b 3' RACE analysis showing the alternative transcripts. $P$ Patient sample, $N$ normal individual, C1 RT - from patient sample, $C 2 \mathrm{RT}$ - from sample of normal individual. c Scheme illustrating the two transcripts with the alternative polyA signals.

d Chromatograms and sequences from transcript I and II. The hexanucleotide motifs of the polyA signals are underlined a

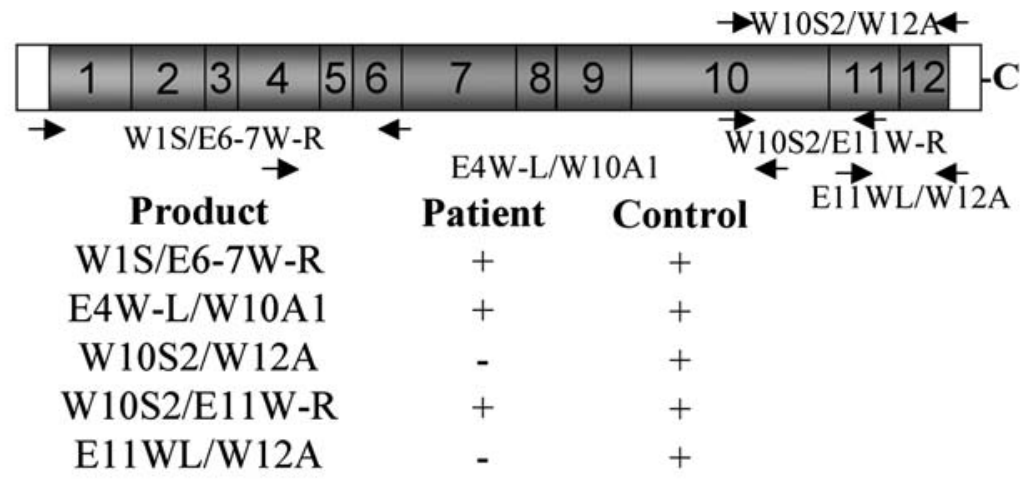

b

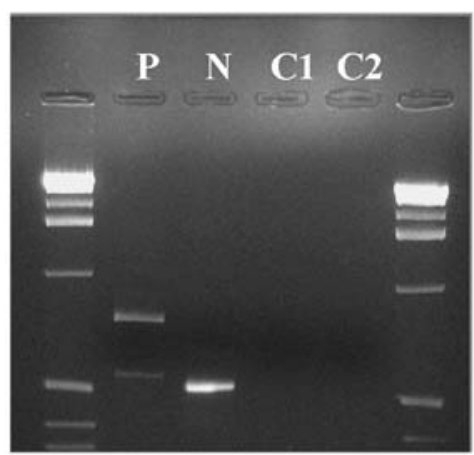

C
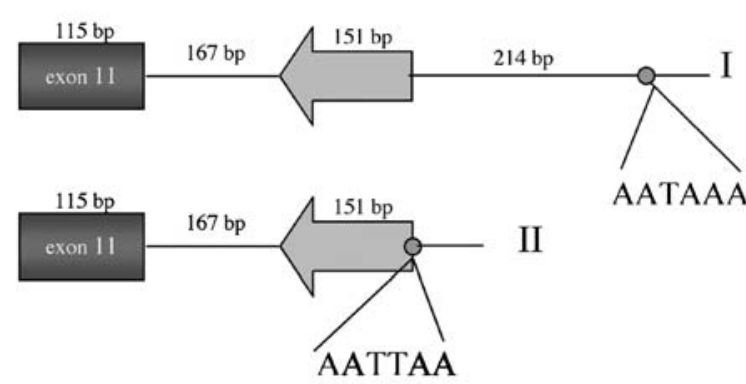

AATAAA d $_{\text {I }}$
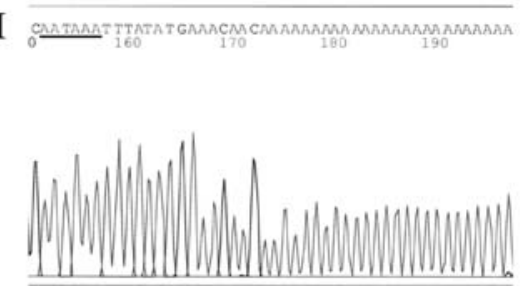

....CCGGTG.... TTAGCC.... TTATAATTAACGTG GTGAAACCCCATCATCTCTACTAAAAATACAAAAATTA GCCAGGTGTGGTGGCACACGCCTGTAATCCCACCTACT CAGGAGGCAGAGGCAGGAAAATCGCTTGAACCCGGGAG GCAGAGGTTGCAGTGAACCGAAATCACGCCACTGCACT CCAGCCTGGGTGACAGAGTGAGACTCTGTCTCAAAAAA AAAATATTATATTTGTACCCAATAAATTTATATGAAAC $\operatorname{AAC}(A) n$

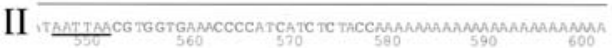
....CCGGTG. . TTAGCC..... TTATAATTAA CGTGGTGAAACCCCATCATCTCTAC (A)n

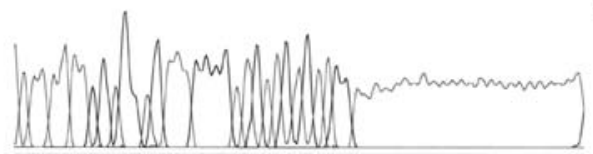

may be nonfunctional, due to the important domains affected. It is known that the $\mathrm{C}$-terminal region of WASP, coded by exon 11 and 12, contains a verprolin central acidic (VCA) domain that interacts with

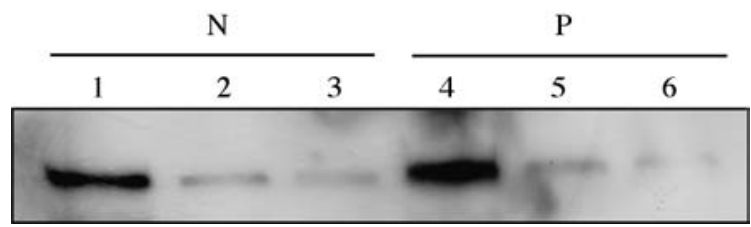

Fig. 3 Anti-WASP Western blot analysis from peripheral blood mononuclear cells. $N$ normal individual, $P$ patient. Lysates from $10^{6}$ cells were loaded onto lanes 1 and 4 , and serial dilutions were loaded onto lanes 2, 5 and 3,6 monomeric actin and the nucleator actin complex Arp2/ 3 (Orange et al. 2004). The mutation in the WAS patient affecting the VCA domain may result in the impairment of such interactions, strongly affecting actin polymerization and leading to alterations in the biological processes in which WASP is involved. As a consequence, the reported mutation would appear to correlate with the severe phenotype observed in the WAS patient.

Acknowledgements This work has been supported by Fundació La Marató de TV3, contract grant number: 99/3510. N.A. is supported by a contract from the Fondo de Investigación Sanitaria. We are grateful to the Servei de genomica of the Universitat Pompeu Fabra. We would also like to thank Maria Piqué and Raúl Mendez for providing us with RACE primers. 


\section{References}

Andreu N, Carreras C, Prieto F, Estivill X, Volpini V, Fillat C (2003) Identification and characterization of a novel splice-site mutation in a patient with Wiskott-Aldrich syndrome. J Hum Genet 48:590-593

Andreu N, Pujol-Moix N, Martinez-Lostao L, Oset M, MunizDiaz E, Estivill X, Volpini V, Fillat C (2003) Wiskott-Aldrich syndrome in a female with skewed $\mathrm{X}$-chromosome inactivation. Blood Cells Mol Dis 31:332-337

Beaudoing E, Freier S, Wyatt JR, Claverie JM, Gautheret D (2000) Patterns of variant polyadenylation signal usage in human genes. Genome Res 10:1001-1010

Brickell PM, Katz DR, Thrasher AJ (1998) Wiskott-Aldrich syndrome: current research concepts. Br J Haematol 101:603-608

Colgan DF, Manley JL (1997) Mechanism and regulation of mRNA polyadenylation. Genes Dev 11:2755-2766

Derry JM, Ochs HD, Francke U (1994) Isolation of a novel gene mutated in Wiskott-Aldrich syndrome. Cell 78:635-644

Devriendt K, Kim AS, Mathijs G, Frints SG, Schwartz M, Van Den Oord JJ, Verhoef GE, Boogaerts MA, Fryns JP, You D, Rosen MK, Vandenberghe P (2001) Constitutively activating mutation in WASP causes X-linked severe congenital neutropenia. Nat Genet 27:313-317

Fillat C, Espanol T, Oset M, Ferrando M, Estivill X, Volpini V (2001) Identification of WASP mutations in 14 Spanish families with Wiskott-Aldrich syndrome. Am J Med Genet 100:116-121

Frohman MA (1990) RACE: Rapid amplification of cDNA ends. In: Innis MAGD, Sninsky JJ, White TJ (eds) PCR protocols: a guide to methods and applications. Academic, New York, pp $28-38$

Hagemann TL, Mares D, Kwan S (2000) Gene regulation of Wiskott-Aldrich syndrome protein and the human homolog of the Drosophila Su(var)3-9: WASP and SUV39H1, two adjacent genes at Xp11.23. Biochim Biophys Acta 1493:368-372

Imai K, Morio T, Zhu Y, Jin Y, Itoh S, Kajiwara M, Yata J, Mizutani S, Ochs HD, Nonoyama S (2004) Clinical course of patients with WASP gene mutations. Blood 103:456-464
Inoue $\mathrm{H}$, Kurosawa $\mathrm{H}$, Nonoyama $\mathrm{S}$, Imai $\mathrm{K}$, Kumazaki $\mathrm{H}$, Matsunaga T, Sato Y, Sugita K, Eguchi M (2002) X-linked thrombocytopenia in a girl. Br J Haematol 118:1163-1165

Jin Y, Mazza C, Christie JR, Giliani S, Fiorini M, Mella P, Gandellini F, Stewart DM, Zhu Q, Nelson DL, Notarangelo LD, Ochs HD (2004) Mutations of the Wiskott-Aldrich syndrome protein (WASP): hotspots, effect on transcription, and translation and phenotype/genotype correlation. Blood 104:4010-4019

Lutskiy MI, Sasahara Y, Kenney DM, Rosen FS, RemoldO'Donnell E (2002) Wiskott-Aldrich syndrome in a female. Blood 100:2763-2768

Martin F, Toscano MG, Blundell M, Frecha C, Srivastava GK, Santamaria M, Thrasher AJ, Molina IJ (2005) Lentiviral vectors transcriptionally targeted to hematopoietic cells by WASP gene proximal promoter sequences. Gene Ther 12:715-723

Ochs HD, Rosen FS (1999) Primary immunodeficiency diseases. In: Ochs HDSC, Puck JM (eds) Primary immunodeficiency diseases: a molecular and genetic approach, vol 24. Oxford University Press, Oxford, pp 292-305

Orange JS, Stone KD, Turvey SE, Krzewski K (2004) The WiskottAldrich syndrome. Cell Mol Life Sci 61:2361-2385

Parolini O, Ressmann G, Haas OA, Pawlowsky J, Gadner H, Knapp W, Holter W (1998) X-linked Wiskott-Aldrich syndrome in a girl. N Engl J Med 338:291-295

Schindelhauer D, Weiss M, Hellebrand H, Golla A, Hergersberg M, Seger R, Belohradsky BH, Meindl A (1996) Wiskott-Aldrich syndrome: no strict genotype-phenotype correlations but clustering of missense mutations in the amino-terminal part of the WASP gene product. Hum Genet 98:68-76

Snapper SB, Rosen FS (1999) The Wiskott-Aldrich syndrome protein (WASP): roles in signaling and cytoskeletal organization. Annu Rev Immunol 17:905-929

Zhao J, Hyman L, Moore C (1999) Formation of mRNA 3' ends in eukaryotes: mechanism, regulation, and interrelationships with other steps in mRNA synthesis. Microbiol Mol Biol Rev 63:405-445

Zhu Q, Zhang M, Blaese RM, Derry JM, Junker A, Francke U, Chen SH, Ochs HD (1995) The Wiskott-Aldrich syndrome and $\mathrm{X}$-linked congenital thrombocytopenia are caused by mutations of the same gene. Blood 86:3797-3804 